almeno parziale - alla tradizione novellistica, tanto da consentire all'A. di ottenere un profilo tutto sommato coerente dei lettori tardi di quei testi (come Mirabeau) - ed è il capitolo Traductions et adaptations à la veille de la Révolution. Ecatonfilea, Deifira et leur lecteurs, p. 173 s. L'addizione di Simiae al gruppo dei testi dell'Alberti meno "fortunato", la conferma del Naufragus e la sostanziale cancellazione dell'Istorietta (viziata peraltro dalla storia di quel vistoso falso del Bonucci che nel ms. Cerchi 16 dell'Archivio di Stato di Firenze), sino all'importante restituzione di testo + figura del $17^{\circ}$ ex ludis rerum mathematicarum sono alcune delle oramai note quanto decisive acquisizioni di F. nel corso del suo lungo tirocinio di filologo e lettore di testi; laddove con la terza parte dell'opera i differenti modi di una lettura tematica centrata sui concetti di famiglia e di donna sullo sfondo del quadro borghese della ricordanza portano sì a rilevare un certo qual distacco albertiano dalle radicate concezioni ecclesiastiche in argomento, ancora giusta «la lutte entre un principe d'individualisme, qui est également culte personnel d'une ratio morale, et un besoin de reconnaissance sociale, d'utilité, d'élan vers les autres, d'une action plus concrète», ma anche consentono di leggere la susseguente, albertianamente misogina condanna della figura della donna nella chiave dello schema del disordine umano-irra- zionale da sempre combattuto dall'umanista. Lo stesso recupero interpretativo della Dissuasio Valerii, operato da F. nel capitolo De Gauthier Map à Leon Battista Alberti. Sur la misogynie comme remedium amoris et cura sui, riesce a sigillare a dovere un poderoso insieme di ricerche come questo, integrandone liminarmente il significato con le parole che riassumono la coerenza e del libro e delle sue singole sezioni nei confronti della "stagione del dialogo" consegnataci da uno dei giacimenti più vasti della cultura albertiana: «La transposition en volgare ou le remaniement de la Dissuasio Valerii qu'entend constituer et constitue de fait la Risposta albertienne se développe donc selon deux directions principales qui peuvent être définies comme une laïcisation radicale du texte d'une part et, de l'autre, comme une réecriture qui semble marquée en profondeur par l'émergence de valeurs et d'idéaux humanistes - pour ne pas dire albertiens - tels que l'autonomie de la culture, le besoin de la recherche historique ou érudite, la nécessité des arts, de l'investigation philosophique». Giacimento che F. ha avuto il merito di studiare nel tempo così nel suo complesso come nelle singolari esecuzioni storiche, con una lucidità di sguardo costantemente nutrita dalla ratio philologica che si può dire in tutto ammirevole.

Marcello Ciccuto

\title{
Giacomo LEOPARDI
}

\section{Cants,}

traducció i notes de Narcís Comadira, edició bilingüe, Barcelona: Edicions 62-Empúries, 2004.

Siamo particolarmente contenti di presentare ai nostri lettori questa straordinaria versione dei Canti di Leopardi a cura del poeta catalano Narcís Comadira. L’opera maggiore del poeta recanatese è finalmente approdata al catalano grazie alla versione di uno dei più valenti traduttori e poeti contemporanei. Sembra sia arrivata l'ora che una delle voci più intense della poesia universale di tutti i tempi, ma anche una delle più incomprese dalla critica in generale, giunga comprensibile ai 
lettori, se non altro a quelli dell'ambito catalano, e sia da essi accettato nella sua radicalità. Ricordiamo in questo senso che la storia del leopardismo, certamente nella Penisola iberica, ma in genere anche nel resto del mondo, si riassume nella celebrazione delle sue forme poetiche e nel simultaneo rifiuto del suo pessimismo nichilista, accettabile soltanto nella considerazione delle contingenze fisiche e familiari che hanno fatalmente tribolato la sua esistenza. Ė evidente che ci sono state molte eccezioni, Pirandello in testa, rispetto a questo atteggiamento, ma l'approccio a questo artefice del pensiero-poetante moderno è stato condizionato a lungo dal «ma» indicante la non accettazione sia delle sue forme troppo "complesse» sia e soprattutto della sua filosofia del nulla.

In tale senso in ambito ispanico sono usciti studi e traduzioni che hanno finalmente reso giustizia alla "diversità» del poeta di Recanati e alla sua forza poetico-filosofica. I capitoli dedicati al titanismo leopardiano all'interno dell' Heroe y el Único di Rafael Argullol, l'antologia dello Zibaldone in spagnolo (1990) con un prologo di questo stesso autore, la versione catalana delle Operette morali a cura di R. Arqués e, soprattutto, la traduzione completa dei Canti (1998) a cura di María de las Nieves Muñiz corredata da un imponente commento, sono indicativi di questo cambiamento di ottica, anche se c'è ancora qualche poeta spagnolo, responsabile di una non troppo autorevole versione completa dei Canti - nel complesso troppo edulcorata - (mi riferisco a quella di Antonio Colinas, soprattutto alla prima edizione 1980, poi rivista nel 1998), che parte ancora dall'idea che Leopardi è un poeta e un pensatore eccessivo, un mistico ateo, un Fray Luis de León senza fede cristiana.

La traduzione catalana di Comadira dei Canti di Leopardi rappresenta l'ultima tappa di un lungo cammino intrappeso un secolo e mezzo fa da molti autori catalani per avvicinare i testi leopardiani alla lingua catalana, il quale, pur annoverando varie traduzioni di Carner, Garcés ed altri, registra soltanto una sola traduzione completa dei Canti, quella di Alfons Maseras (Barcellona, 1938). Maseras, attento italianista e entusiasta leopardista, ci ha dato, comunque, una versione in cui il tentato rispetto della metrica e della forma non va di pari passo con quello per lo stile e per il pensiero leopardiani - cosa già dimostrata altrove. Curiosamente significativo risulta il prologo in cui egli rivendica un improponibile Leopardi, lottatore per le patrie libertà (affine in qualche modo alle prime interpretazioni del «progressismo politico» leopardiano da parte di Luporini nel suo Leopardi progressivo), comprensibile soltanto se ricordiamo che gli anni in cui scrive sono quelli della guerra civile spagnola.

Di ben altro spessore è la versione di Comadira, frutto di parecchi anni di riflessione e di una frequentazione assidua dell'intera opera leopardiana, come testimoniano le diverse traduzioni parziali che arricchiscono l'antologia Poesia italiana del 1985, le conferenze e gli articoli che il poeta catalano ha dedicato all'autore italiano, nonché il suo racconto di un viaggio a Recanati, che oggi, insime a altri studi sul recanatese, possiamo trovare riuniti in Lànima dels poetes (Barcellona, 2002, p.132-146).

Questa versione definitiva dei Canti, con il testo italiano a fronte, è corredata da un prologo e dalle note del traduttore in cui si affrontano alcuni problemi di traduzione e, innanzitutto, il rapporto intertestuale esistente tra i componimenti e tra gli i Canti, lo Zibaldone e le Operette morali. Nel prologo si insiste particolarmente sul rapporto del poeta marchigiano con la sua famiglia e con il paese che gli ha dato i natali, e viene riportato un brano della lettera che Leopardi scrisse a suo padre nel 1819, palese testimonianza del conflitto esistente tra i due, nel quale forse lo stesso Comadira avrà rivissuto quello da lui stes- 
so descritto nei componimenti di Terra natal (1978) e di Álbum de família (1980) («I Girona mesquina: Sanctus, Sanctus», de La visita del jerarca i «I tu, papà, gran cabronàs, ¿què hi feies / a dalts de la tribuna, si no eres feixista", de 4 de febrer de 1945). Inoltre Comadira sottolinea la differenza stilistica e tonale fra le "canzoni» e gli «idilli» leopardiani come due varianti dalla cui sintesi uscirà il "pensiero poetante»: «el pensament que es va configurant mentre el discurs líric es teixeix». In queste pagine iniziali Comadira, oltre a informarci che la sua fatica di traduzione di Leopardi era iniziata nel 1985, si soffferma in special modo a spiegare che il suo sistema traduttorio è rimasto sostanzialmente invariato nel tempo, benché la sua esperienza e la sua frequentazione del recanatese andassero appianando strade impervie e superando ostacoli prima insormontabili. Se da tempo il poeta catalano aveva trovato nell'adesione alla forma e allo stile dell'originale il meccanismo essenziale del suo modo di tradurre, oggi ha individuato nel recente libro di Eco, Dire quasi la stessa cosa. Esperienze di traduzione (2003) la teorizzazione di questa procedura, secondo la quale ogni traduttore deve tentare una duplice e simultanea operazione: dire «quasi la stessa cosa» e dirla "quasi allo stesso modo».

Le varianti di traduttore che si sono accumulate in questi anni costituiscono la prova di questo intenso infaticabile e costante lavoro con cui il traduttore mirava a rendere la sua versione il più possibile vicina e equivalente all'originale. In questo senso è emblematico lo studio delle varianti della traduzione dell'Infinito e in special modo del primo verso, quello più relaborato: dal calco iniziale «em fou car» si è passati al colloquiale "he estimat», mentre l'"eixorc turò" con cui il traduttore aveva esordito (forse sotto la suggestione della recente versione catalana di The waste Land, «la terra eixorca») è diventato "turó desert» nell'ultima versione. I ripensamenti riguardano anche «l'esguard em priva» del verso 3 che appare sia nella versione iniziale sia in quella definitiva, dopo essere stato reso per un lungo periodo intermedio come «exclou la mirada». Ragioni metriche devono aver spinto invece il traduttore a snellire il filto sistema dei deittici della poesia in questione, riducendo in particolare i pronomi dimostrativi quella, quello ai pronomi personali «ella»e "el» rispettivamente; sistema che ha un ruolo fondamentale in questo componimento giacché da essi dipende l'annullamento della distinzione tra soggetto/oggetto, finito/infinito, spazio/tempo.

L'altissima prova di armonia musicale, linguistica e di significato rappresentata dalla traduzione comadiriana dei Canti è anche e soprattutto un gioco di equilibrio e di compensazione della richissima lingua artistica del recanatese, nella quale primeggia l'iperbato. Questo elemento, che non sempre è stato osservato dai traduttori, relegato spesso a caratteristica propria di un periodo ormai tramontato della poesia oppure a complicazione innecessaria, è preso seriamente in considerazione dal nostro traduttore, $\mathrm{e}$ sempre con fine eleganza, ora abolendolo dove la lingua o la cultura d'arrivo sembrano meno disposte ad accettarlo (cioè nel caso dell'aggettivo davanti al nome), ora mantenendolo dove questa figura è parte essenziale del discorso poetico, come è il caso, riuscitissimo della, versione di $A$ se stesso (A si mateix), in cui è mantenuta tutta la complessità sintattica dei frastagliati versi della lirica leopardiana, così intensamente e serenemante disperata. Ma questo è soltanto uno dei tanti momenti di piena felicità poetica che Comadira ha saputo realizzare.

Non per nulla la poesia leopardiana è stato in tutti questi anni per Comadira un punto di riferimento formale oltre che ideologico così costante da lasciare tracce indelebili nella sua poesia, basti citare la Oda a Pep Guardiola nella quale non si può non sentire riecheggiare quella leopardiana $A$ un vincitore nel pallone. Ma lo 
stesso poeta in un recente convegno dedicato a Leopardi ha precisato le ragioni per le quali si è sentito da sempre legato al "cigno di Recanati»: a) il suo sguardo limpido, scevro da fingimenti; b) il rifiuto di una realtà fondata sull'elogio, che nasconde la codardia; c) il rapporto tra destino recanatese e destino catalano: due carce- ri da cui entrambi i poeti tentato invano di liberarsi; d) la vita umana votata al nulla; e) la «felicità leopardiana del composar poesies», malgrado tanta negatività. Leopardi: ancora una vera fonte d'ispirazione per la poesia moderna.

\section{Rossend Arqués}

\section{Italo SVEVO}

La conciencia de Zeno,

Introducción de Elisa Martínez Garrido, Traducción y notas de Mercedes Rodríguez Fierro, Madrid: Gredos, 2002

Come ha osservato Debenedetti, nel suo capitale Il romanzo del Novecento sia Zeno che Svevo, piú che raggiungere il successo, ne sono raggiunti; tardi, fuori tempo e a sorpresa. Ora da questo successo, tardo, senile, autunnale, La coscienza di Zeno ha raggiunto lo stabile e pressoché unanime statuto di capolavoro della modernità, come non mancava di osservare nel suo originale e al solito di intelligente e provocatoria iconoclastia, Beradinelli nell'ultimo dei volumi einaudiani dedicati al romanzo; l'eredità su Pontiggia, narratore psicologistico e aforisitico di stratificati grovigli psichici, e il Maggiani de Il coraggio del Pettirosso, per chiamare in causa due rilevanti autori del tardo secondo Novecento stanno lì a dimostrarlo. Lo dimostra pure la pervicace e sottile fascinazione che l'umorismo subdolo del narrato di Zeno esercita su Robbe-Grillet, Philip Roth e Saul Bellow. In Spagna costituiscono dei capitoli importanti il saggio di Anna Dolfi nell'edizione di Cátedra del 1998, quello di Muñiz nel volume miscellaneo curato dalla stessa Dolfi e l'intervento di Albertocchi nel numero precedente di questa rivista. L'edizione che qui si recensisce curata e tradotta rispettivamente da Elisa Martínez Garrido e Mercedes Rodríguez Fierro, mette a disposizione del lettore una nuova edizione di questo fondante ed insidioso classico della nostra modernità. Seguendo nell'ordine del nostro discorso l'esposizione dei punti affrontati dalla solida introduzione, pur nella non completa originalità delle argomentazioni (di fatto si alimenta dei notevoli studi di Anna Dolfi e Mario Lavagetto), ci si può soffermare sulla buona contestualizzazione dell'esperienza narrativa di Svevo: alla triestinità l'autrice dell' introduzione riconduce alcune caratteristiche salienti della fisionomia intellettuale dell'autore, l'ironia, lo scetticismo conoscitivo, la duplicità contraddittoria. Brevi paragrafi dedicati alla biografia dell'autore rilevano come la letteratura occupi un luogo antagonisitico rispetto alla legge del padre, ovvero sia lo spazio, che in chiave psiconalitica, viene definito della rappresentazione del desiderio, dell'immaginario. Particolare interesse ha la lettura del personaggio sveviano attraverso il confronto con il Nitti e il Brentani dei romanzi anteriori, confronto da cui si deduce una crescita nel senso dell'autocoscienza e dell'ironia. Da qui prende abbrivio la parte più cospicua dell'introduzione, i cui punti cardinali riassumo a seguire. Nella struttura psicologica della rivalità viene individuato il centro propulsore di tutto lo sviluppo narrativo del testo. Ne consegue il fatto che l'opzione per l'oratio obliqua dello stile indiretto libero e la rinuncia al monologo interiore venga interpretata come risultato testuale, parafraso, che promana dalla preponderanza della coscienza morale e dal 